\title{
Grandes écoles et universités: un système de formation à harmoniser
}
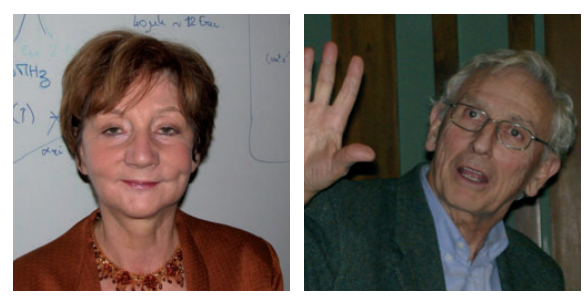

La coexistence des grandes écoles et des universités crée en France une divergence de culture et un cloisonnement de carrière pour les cadres scientifiques, qui handicapent la nécessaire fluidité entre les métiers de la science. Les écoles d’ingénieurs et leurs classes préparatoires (CPGE) se sont multipliées depuis vingt ans et les meilleurs bacheliers choisissent massivement l'orientation vers les filières sélectives. En licence de physique, le nombre des étudiants est en baisse presque partout et leur disparité de niveau par rapport aux élèves de CPGE est flagrante. De plus, les formations longues du système universitaire ont des débouchés limités car les entreprises préfèrent généralement embaucher les ingénieurs sortis d'écoles plutôt que des docteurs d'université.

La première urgence nous semble être d'augmenter le flux de bons étudiants à l'université. La qualité de la formation fournie en licence est d'importance primordiale. L'encadrement des étudiants est certainement à améliorer, avec un contrôle continu décourageant l'amateurisme et des objectifs précis en termes d'acquisition des connaissances et de savoir-faire, corrigeant l'émiettement résultant du LMD. Une bien meilleure information comparative en terminale sur les cursus universitaires et les débouchés des différentes filières augmenterait la visibilité du champ des métiers possibles.

L'amélioration de la vie sur les campus est un autre élément pour y attirer plus de jeunes, avec un service d'accueil chaleureux, une vie culturelle sur place, des lieux de rencontre, des locaux ouverts tard le soir, et évidemment des logements décents en nombre suffisant - ce qui implique, il est vrai, des moyens dont manquent cruellement les établissements, en dépit de la future manne du plan Campus, fortement médiatisée mais finalement assez modeste. Tout ceci serait d'ailleurs de nature à augmenter l'attrait de nos universités vis-à-vis des étudiants étrangers, pour lesquels on devrait aussi penser à des cours intensifs de langue française, des « jobs " étudiants et des enseignements en anglais au niveau du master et du doctorat.

De plus, nous pensons souhaitable que s'instaure une vraie réflexion sur la sélection à l'entrée des universités. II nous paraît évident qu'on ne peut pas vouloir développer des universités d'élite pour la recherche et continuer à y accueillir tous les bacheliers sans tri. Afin de développer dans les universités une saine émulation avec les CPGE, pourquoi ne pas permettre aux universités de mettre en place dans leurs domaines d'excellence des programmes ambitieux dont le recrutement serait inévitablement sélectif ? Avec des chercheurs de haut niveau comme enseignants, les étudiants fortement motivés formeraient ainsi un vivier de jeunes scientifiques de premier plan, aussi bien pour la recherche que pour la $\mathrm{R}$ et $\mathrm{D}$ de niveau supérieur dans les entreprises.

Enfin, nous pensons qu'on peut faire progressivement évoluer le système pour favoriser le rapprochement entre les CPGE et les universités. II est en effet dommage que le succès des CPGE prive leurs élèves du contact avec la recherche. Une osmose entre les formations devrait être établie, sous forme d'accueil des étudiants de CPGE au sein des laboratoires de recherche dans les universités, par exemple pour des stages et tout particulièrement pour la préparation de leurs travaux d'initiative personnelle encadrés (les TIPE). Les professeurs de CPGE et ceux des universités se verraient offrir des possibilités de croiser leurs services d'enseignement et des détachements seraient facilités. Un tel rapprochement serait l'occasion de rénover les programmes des CPGE en physique et chimie, très encyclopédiques et trop tendus, au profit d'un approfondissement disciplinaire et d'une participation plus personnelle des élèves.

En résumé, nous avançons ici quelques suggestions pour rapprocher les formations et limiter la monoculture de nos cadres scientifiques, issus presque exclusivement des grandes écoles, afin que les jeunes étudiants puissent choisir leur orientation et trouver les conditions correspondant au mieux à leur motivation.

Michèle Leduc et Roger Maynard 